\title{
Diffraction-limited coherent wake emission
}

\author{
E. Porat $\odot,{ }^{1,2,{ }^{*}}$ H. Yehuda, ${ }^{1}$ I. Cohen $\odot,{ }^{1}$ A. Levanon,,${ }^{1}$ and I. Pomerantz ${ }^{1}{ }^{1}$ \\ ${ }^{1}$ The School of Physics and Astronomy, Tel Aviv University, Tel Aviv 69978, Israel \\ and Center for Light-Matter Interaction, Tel Aviv University, Tel Aviv 69978, Israel \\ ${ }^{2}$ Department of Applied Physics, Soreq Nuclear Research Center, Yavne 81800, Israel
}

(Received 9 March 2021; accepted 5 August 2021; published 7 September 2021)

\begin{abstract}
The plasma mirror is a relativistic optical element that under certain irradiation conditions generates intense attosecond extreme-ultraviolet light pulses, in a process known as coherent wake emission (CWE). CWE has been previously characterized by its high spatial divergence, originating from an intrinsic intensity-dependent phase accumulation. In this Letter, we show that the transverse variations of the plasma expansion can completely cancel the CWE intrinsic phase. Accordingly, we experimentally demonstrate nearly diffraction-limited CWE with unprecedented divergence, under $6 \mathrm{mrad}$. We validate our analytical model with particle in cell simulations. This understanding facilitates the development of plasma mirrors as applicable ultraviolet light sources.
\end{abstract}

DOI: 10.1103/PhysRevResearch.3.L032059

Chirped-pulse amplification (CPA) technology made possible the realization of intense laser fields, in which oscillating electrons reach nearly the speed of light. This so-called relativistic optics regime [1] prevails at an intensity threshold of about $10^{18} \mathrm{~W} \mathrm{~cm}^{-2}$ for typical $800 \mathrm{~nm}$ central wavelength laser systems. Examples are plasma-based optical elements that act as nonlinear media [2], and as optical switches [3] and relativistic shutters [4] with femtosecond rise times.

The simplest plasma-based optical element is the plasma mirror (PM) [3]. When an intense laser pulse is focused on a polished surface, the target becomes strongly ionized and forms plasma that reflects the incident pulse. If the incident pulse is ultrashort, the plasma does not expand significantly, and the laser pulse is specularly reflected.

Despite their simplicity, PMs present highly nonlinear laser-plasma dynamics, manifested by the generation of synchronized attosecond light [5] and electron bursts [6]. These are of great interest to ultrafast science, because the availability of ultrashort, coherent pulses in the extreme-ultraviolet (XUV) region is a prerequisite for many applications, such as attosecond spectroscopy [7], biological imaging [8], and material sciences [9].

In the frequency domain, the XUV emission manifests in a discrete harmonic spectrum of the fundamental laser wavelength. These so-called surface harmonics have been established to originate from distinct types of plasma dynamics, divided into two regimes of laser intensity: relativistic processes driven by ultrahigh intensities of over $10^{19} \mathrm{~W} \mathrm{~cm}^{-2}$, such as the relativistic oscillating mirror [10] and coherent

\footnotetext{
*elkanaporat@mail.tau.ac.il

Published by the American Physical Society under the terms of the Creative Commons Attribution 4.0 International license. Further distribution of this work must maintain attribution to the author(s) and the published article's title, journal citation, and DOI.
}

synchrotron emission [11,12], and a subrelativistic process known as coherent wake emission (CWE) which prevails at moderate intensities above $10^{16} \mathrm{~W} \mathrm{~cm}^{-2}$ [13]. Relativistic processes are often considered more promising for applications, due to their higher bandwidth and efficiency [14]. However, these attributes are mainly manifested in the ultrarelativistic regime, approachable only by petawatt-class laser systems $[15,16]$. CWE on the other hand, with its lower intensity requirement, is easily accessible by university-scale laser systems and its efficiency of about $10^{-4}$ (at $80 \mathrm{~nm}$ wavelength) [13] surpasses typical gas-based harmonic generators.

A main drawback that limits the applicability of CWE as an XUV light source is its excessive beam divergence, which makes refocusing the attosecond pulses extremely challenging. The excessive divergence is associated with intrinsic phase variation across the laser focal spot $[17,18]$. In CWE, vacuum-accelerated Brunel electrons [19] collide in the sharp density gradient of the plasma surface and induce highfrequency plasma waves. After a short evolution time, when a local phase-matching condition is met, these waves reemit XUV radiation [13]. The amount of phase accumulated during this three-step process depends on the local laser intensity and on the local plasma scale length [20]. Phase induced by spatiotemporal intensity variations was shown to broaden the spectrum and the spatial divergence of CWE [17,18,20-23].

In surface harmonics experiments, preplasma is often engineered by introducing a loosely focused auxiliary prepulse (APP) beam [24,25]. The rather uniform illumination of the APP at the focal spot area of the main beam justifies neglecting the effect of lateral variations of the plasma scale. We show here, however, that when preplasma is formed by native precursor light (NPL) of the beam itself, lateral variations of the plasma scale are fundamental to the harmonic beam divergence.

In this Letter we first introduce a modification to the CWE emission model of Ref. [21], to account for these spatial variations. Next, we present experimental data supporting 


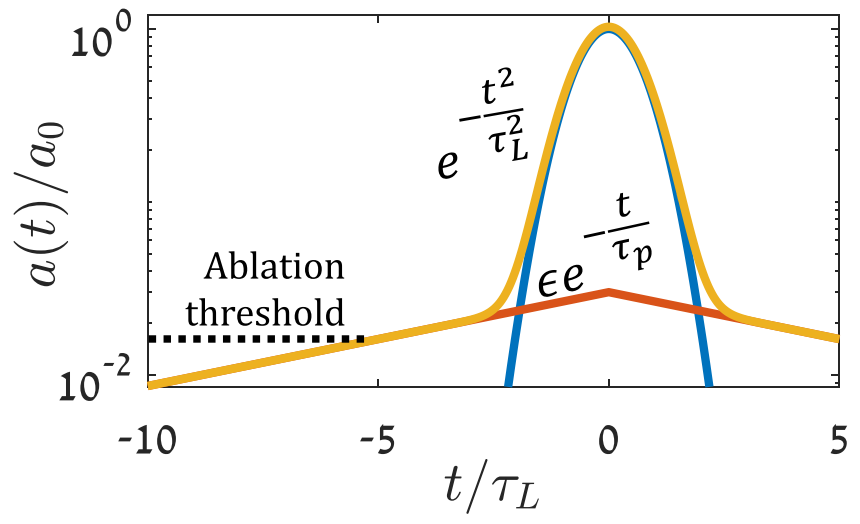

FIG. 1. An illustration of the laser intensity temporal evolution (yellow) considered in our derivation. An exponentially rising pedestal (red) is followed by an intense ultrashort pulse, e.g., Gaussian shaped (blue). Ablation is assumed to initiate during the pedestal phase.

our model, in which CWE beams are controlled to become highly collimated, with nearly diffraction limited divergence. We conclude with a numeric particle in cell (PIC) study that validates the results.

To model the local plasma expansion across the focal spot, we determine the plasma scale length by time integrating the plasma velocity until the peak of the pulse (at $t=0$ ):

$$
L(\boldsymbol{r}, 0)=\int_{-t_{i}(\boldsymbol{r})}^{0} C_{s}(\boldsymbol{r}, t) d t,
$$

where $C_{s}$ is the ion-acoustic velocity and $-t_{i}$ is the initiation time of the plasma motion. Under the assumptions of instantaneous thermalization and monotonically increasing intensity, the ion-acoustic velocity under subrelativistic irradiation can be estimated to scale as $C_{s} \approx C_{0} a(\boldsymbol{r}, t)$ [26], with $a^{2}=$ $I \lambda^{2} /\left(1.38 \times 10^{18} \mathrm{~W} \mathrm{~cm}^{-2} \mu \mathrm{m}^{2}\right)$ being the local value of the normalized vector potential. As illustrated in Fig. 1, in modern high-power amplification schemes [27,28] the temporal intensity profile can be described as being composed of a low-intensity, slowly rising "pedestal," and a high-intensity ultrashort main pulse:

$$
a(\boldsymbol{r}, t)=a_{0} \eta\left(\boldsymbol{r} / w_{L}\right)\left[P\left(t / \tau_{L}\right)+\epsilon \exp \left(-t / \tau_{p}\right)\right],
$$

where $\eta$ is the radial intensity distribution of a spot with radius $w_{L} ; P$ is the temporal evolution of the main pulse with duration $\tau_{L}$, e.g., a Gaussian; $\epsilon \ll 1$ is the amplitude of the pedestal normalized at $t=0$; and $\tau_{p}>\tau_{L}$ is its time constant.

The substitution of Eq. (2) separates Eq. (1) into two parts, for the main pulse and the pedestal, that can be solved separately. We assume the plasma expansion initiates during the pedestal phase, having $t_{i} \gg \tau_{L}$. Thus, for the main pulse, we can substitute the integral boundary by $t_{i}(\boldsymbol{r}) \rightarrow \infty$, to have

$$
L_{\text {main pulse }}(\boldsymbol{r}) \approx C_{0} a_{0} \eta\left(\boldsymbol{r} / w_{L}\right) \tau_{L} \Pi,
$$

where $\Pi \equiv \int_{-\infty}^{0} P(x) d x$ is an $\mathcal{O}(1)$ shape factor, e.g., $\Pi=$ $\sqrt{\pi} / 2$ for a Gaussian pulse. For the pedestal part, we define $a_{\text {ablation }} \equiv a\left[-t_{i}(\boldsymbol{r})\right]$ and change variables $d t \rightarrow d a$, to obtain

$$
L_{\text {pedestal }}(\boldsymbol{r})=C_{0} \tau_{p}\left[\epsilon a_{0} \eta\left(\boldsymbol{r} / w_{L}\right)-a_{\text {ablation }}\right] .
$$

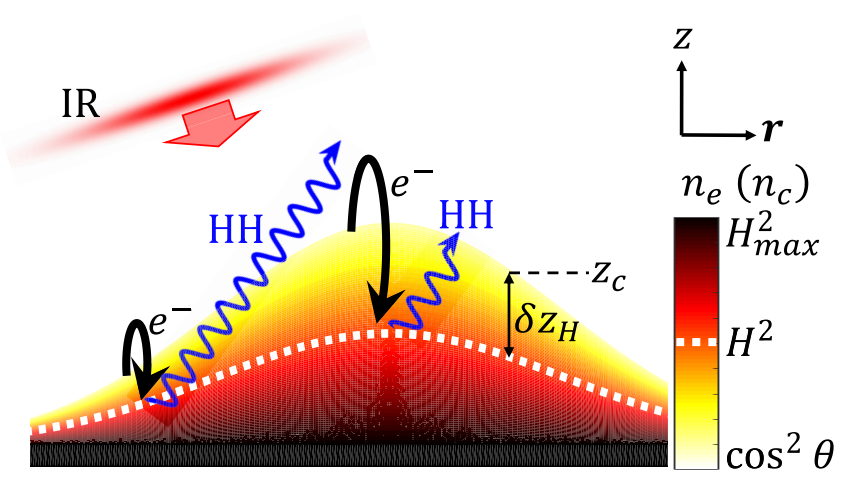

FIG. 2. Schematic illustration of CWE in a three-dimensional (3D) plasma plume. A Gaussian-like plasma (color map) is formed by the IR beam. At the center, a larger plasma scale length forms a shorter optical path, but also a longer path for the Brunel electrons to traverse into the plasma, where they induce $\mathrm{HH}$ emission.

Combining Eqs. (3) and (4) yields a simple solution to the plasma scale length at $t=0$ :

$$
L(\boldsymbol{r}) \approx L_{0}\left[a(\boldsymbol{r})-a_{i}\right],
$$

with the constants $L_{0}$ and $a_{i}$ defined as

$$
\begin{aligned}
a_{i} & \equiv a_{\text {ablation }}\left(\Pi \tau_{\mathrm{L}} / \tau_{p}+\epsilon\right)^{-1}, \\
L_{0} & \equiv \tau_{p} C_{0}\left(\Pi \tau_{\mathrm{L}} / \tau_{p}+\epsilon\right) .
\end{aligned}
$$

The lateral variation of the plasma scale length affects the XUV spatial phase in two ways, illustrated in Fig. 2. First, the optical path of an XUV beamlet generated from an expanded plasma is shortened by [5]

$$
\Delta l=\left(2 z_{c}-\delta z_{H}\right) \cos \theta,
$$

with respect to a laser beam reflected off the unexpanded plasma. Here $z_{c}$ is the position of the laser-plasma interface, and $\delta z_{H}$ the depth at which the $H$ th harmonic is born. Note that $\delta z_{H}$ is accounted for only once, since the harmonics are generated within the plasma. We ignore the plasma dispersion here for the sake of simplicity. In a detailed calculation, the plasma dispersion was found to affect the model parameters by less than $10 \%$.

Assuming an exponential plasma density profile, $n_{e}(z)=$ $n_{0} e^{-z / L-1}, z_{c}$ and $\delta z_{H}$ are given by [23]

$$
z_{c}=2 L \ln \frac{H_{\max }}{\cos \theta}-L, \quad \delta z_{H}=2 L \ln \frac{H}{\cos \theta},
$$

where $\theta$ is the angle of incidence, $n_{c}$ is the critical density of the fundamental laser frequency, and $H_{\max }=\sqrt{n_{0} / n_{c}}$ with $H_{\text {max }} \approx 20$ for fully ionized $\mathrm{SiO}_{2}$ plasma at solid density and $800 \mathrm{~nm}$ laser wavelength. The overall change in the optical path is then

$$
\frac{\Delta l}{\lambda_{H}}=\frac{2 L \cos \theta}{\lambda_{H}}\left[\ln \left(\frac{H_{\max }^{2}}{H \cos \theta}\right)-1\right],
$$

which translates to an intensity-dependent phase term using Eq. (5):

$$
\frac{\varphi_{\Delta l}}{\pi}=-4 \frac{L_{0} \cos \theta}{\lambda_{H}}\left[a(\boldsymbol{r})-a_{i}\right]\left[\ln \left(\frac{H_{\max }^{2}}{H \cos \theta}\right)-1\right] .
$$


A second source for phase accumulation is the duration in which Brunel electrons traverse the plasma and collide at $\delta z_{H}$. This duration was calculated in Ref. [21]:

$$
\frac{t_{e}}{T_{L}}=0.307+0.725\left(\frac{\delta z_{H}}{a \lambda \sin \theta}\right)^{\frac{1}{3}} .
$$

Plugging in Eqs. (5) and (8), and ignoring constant terms, we find the phase term to be

$$
\frac{\varphi_{t_{e}}}{\pi}=1.45 H\left\{\frac{2 L_{0} \ln \frac{H}{\cos \theta}}{\lambda \sin \theta}\left[1-\frac{a_{i}}{a(\boldsymbol{r})}\right]\right\}^{\frac{1}{3}} .
$$

Following Ref. [23], we sum Eqs. (10) and (12), and approximate the focused intensity by $a(\boldsymbol{r}) \approx a_{0}\left(1-r^{2} / w_{L}^{2}\right)$ to find the normalized focusing parameter:

$$
\Psi \equiv \frac{w_{L}^{2}}{\lambda R}=\eta\left(\frac{L_{0}}{\lambda}\right)^{\frac{1}{3}}\left(1-\frac{a_{i}}{a_{0}}\right)^{-\frac{2}{3}} \frac{a_{i}}{a_{0}}-4 \zeta \frac{L_{0}}{\lambda} a_{0},
$$

where $R$ is the phase-front radius of curvature, defined by $\varphi_{H} \approx k_{H} r^{2} / 2 R, \quad \eta=\frac{1.45}{3}\left[2 \ln \left(\frac{H}{\cos \theta}\right) / \sin \theta\right]^{\frac{1}{3}}$, and $\zeta=$ $\cos \theta\left[\ln \left(\frac{H_{\max }^{2}}{H \cos \theta}\right)-1\right]$. Requiring $\Psi=0$ yields the intensity value at which the XUV emission becomes diffraction limited:

$$
a_{0}^{\mathrm{DL}}=\frac{1}{2}\left(\frac{L_{0}}{\lambda}\right)^{-\frac{1}{3}} \sqrt{a_{i} \eta / \zeta} .
$$

To relate the phase-front curvature at the focal plane to the beam divergence at the far field, we assume that the XUV source has a Gaussian profile, with the same waist size $w_{L}$ of the driver laser. The cone angle at the far field of the $H$ th harmonic is then given by [29]

$$
\frac{\theta_{H}}{\theta_{L}}=\frac{1}{H} \sqrt{1+(H \pi \Psi)^{2}},
$$

where $\theta_{L}$ is the effective numeric aperture of the driving laser pulse.

Experiments were carried out using a $20 \mathrm{TW}, 25 \mathrm{fs}, 800 \mathrm{~nm}$ central wavelength laser system at Tel Aviv University. The architecture of the laser, detailed in Ref. [30], is based on picosecond optical parametric chirped-pulse amplification for most of the system gain, followed by a traditional Ti:sapphire power amplifier. A measurement of the laser temporal profile, obtained with a Sequoia third-order autocorrelator [31] and normalized to the focused laser intensity, is shown in Fig. 3(a). The laser system features a temporal contrast better than $10^{11}$ up to $t=-60 \mathrm{ps}$.

In these experiments, the beam was focused using a $16.3 \mathrm{~cm}$ focal length off-axis parabolic mirror onto an optically flat fused-silica substrate, at a $55^{\circ}$ angle of incidence. The beam's numerical aperture was varied using a motorized aperture, which sets the laser intensity both by reducing the pulse energy and by increasing the focal spot size. The focal ratio of the beam was varied in the range of $f / 20-f / 7$, corresponding to focused intensities of $4.5 \times 10^{15} \mathrm{~W} \mathrm{~cm}^{-2}$ to $3 \times 10^{17} \mathrm{~W} \mathrm{~cm}^{-2}$.

A far-field image of the focal spot, taken at $f / 8.6$, is shown in color scale in Fig. 3(b). The spot diameter was measured as $7.9 \mu \mathrm{m}$ [full width at half maximum (FWHM)], corresponding to a focused intensity of $1.3 \times 10^{17} \mathrm{~W} \mathrm{~cm}^{-2}\left(a_{0} \approx 0.25\right)$.

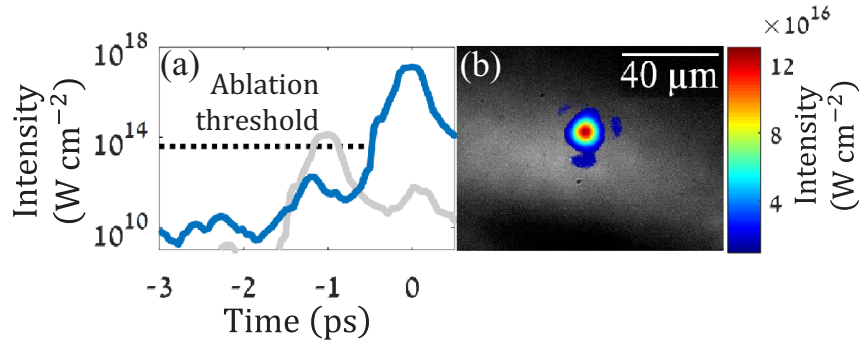

FIG. 3. (a) Temporal profile of the laser at $a_{0} \approx 0.25(I=1.3 \times$ $10^{17} \mathrm{~W} \mathrm{~cm}^{-2}$ ), measured with a third-order autocorrelator and normalized to the intensity at the focus. The APP is represented in light gray, as a weak replica of the main pulse, shifted in time. (b) Spatial intensity distributions of the laser beam (color scale) and the APP (gray scale) at the focus.

The high-harmonics spectra were acquired using a flat-field grazing-incidence XUV spectrograph (HP spectroscopy), with a $2.76 \mathrm{~cm}$ wide back-illuminated $\mathrm{CCD}$ detector (Andor DO936N-M0Z), positioned $1 \mathrm{~m}$ away from the target. A typical spectrogram is shown in Fig. 4(a), for a shot with a normalized intensity of $a_{0}=0.25$. No auxiliary prepulse was introduced in this setup, so that preplasma originates from NPL only. The transverse profile of the 14th harmonic is shown in Fig. 4(b), together with a Gaussian fit that yields a $1 / e^{2}$ radius of $5.8 \pm 0.15 \mathrm{mrad}$.

To verify that the low divergence indeed results from the small transverse preplasma scale, we introduced an APP in a similar method to that of Ref. [18]. A $150 \mu$ m thick mirror was inserted $100 \mu \mathrm{m}$ ahead of the final steering mirror to sample the outer edge of the laser beam. This introduces a wide APP at $t \approx-1$ ps.

A far-field image of the APP beam is shown in gray scale in Fig. 3(b), with a focused intensity of about $10^{14} \mathrm{~W} \mathrm{~cm}^{-2}$, contained within a $150 \times 35 \mu \mathrm{m}^{2}$ ellipse (FWHM). This large lateral extent creates a nearly uniform illumination within the focal spot of the main pulse. The ion-acoustic velocity of the APP plasma is estimated as smaller than $10 \mathrm{~nm} / \mathrm{ps}$ [32], resulting in a plasma scale length of $L_{\mathrm{APP}} \approx \lambda / 100$. The

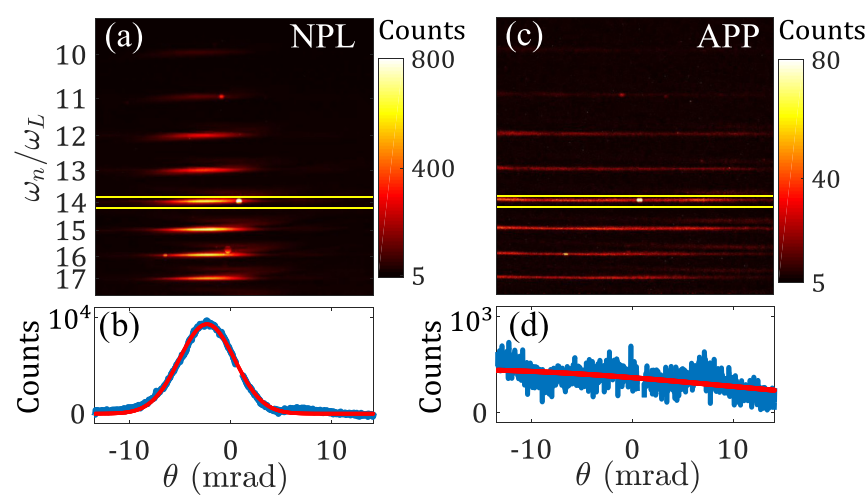

FIG. 4. (a), (c) show raw spectrograms, taken at $a_{0}=0.25$, for the cases of NPL and APP, respectively. (b), (d) show spatial distributions of the H14 intensity, spectrally integrated over the range marked by the yellow lines in (a), (c). Fits to Gaussian form (red) are used to evaluate the angular divergence of each harmonic. 


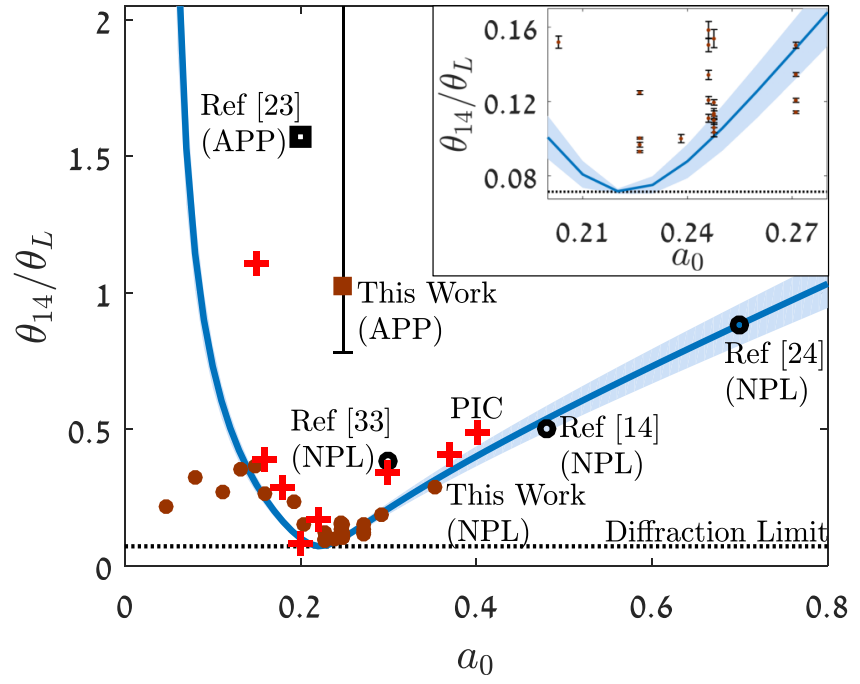

FIG. 5. The beam divergence of H14. Measurements employing an NPL (APP) are marked with circles (squares). Our (previous) work is shown in filled dark-red (open black) markers. The dotted line indicates diffraction-limited divergence. A fit to the analytic model of Eqs. (13) and (15) is shown in solid blue, with $1 \sigma$ uncertainty bounds (shaded). The results of the PIC simulation are marked with crosses. Inset: NPL data points around the diffraction limit intensity. The error bars represent $95 \%$ confidence bounds resulting from the fitting process.

resulting spectrogram for the APP case is shown in Fig. 4(c), for a laser shot taken at the same intensity as the one in Fig. 4(a). A tenfold increase of the XUV divergence is observed by fitting the profile of the 14th harmonic [Fig. 4(d)].

Figure 5 presents the measured divergence of the 14th harmonic normalized to the cone angle of the laser, in an intensity range of $0.05<a_{0}<0.35$. The vertical error bars represent the uncertainty resulting from the fitting procedure. The stability in the range $a_{0}=0.22-0.27$ is found to be smaller than $15 \%$ standard deviation. Our data are compared to APP and NPL results from other works [14,23,24,33], and to our APP control shot. The divergence of our NPL data approaches the diffraction limit (dotted line) at $a_{0} \approx 0.22$, and it is over four times lower than any previously reported data.

To compare the data to our model, we fit Eqs. (13) and (15) in the range of $a_{0}>0.12$, with $a_{i}$ and $L_{0}$ as free parameters. The fit result, which is plotted in blue in Fig. 5, yields

$$
\begin{aligned}
& a_{i}=0.044 \pm 0.002 \\
& \lambda / L_{0}=11.3 \pm 0.6
\end{aligned} \text { and } a_{0}^{\mathrm{DL}}=0.21 .
$$

These parameters correspond to $L \approx \lambda / 70$ near the diffraction limit intensity, which is well within the known range of CWE emission [24].

Interpreting the value of $a_{i}$ according to Eq. (6) for our laser's temporal profile ( $\tau_{L}=25 \mathrm{fs}, \tau_{\text {pedestal }} \approx 200 \mathrm{fs}$, and $\epsilon \ll$ 1 ), we find that the plasma motion was initiated at $\sim 5 \times$ $10^{13} \mathrm{~W} \mathrm{~cm}^{-2}(a=0.005)$, in good agreement with the known ablation threshold of fused silica.

The robustness of our model is demonstrated in Fig. 6, where we compare the predicted evolution of the divergence with the harmonic order to our data, at different laser intensi-

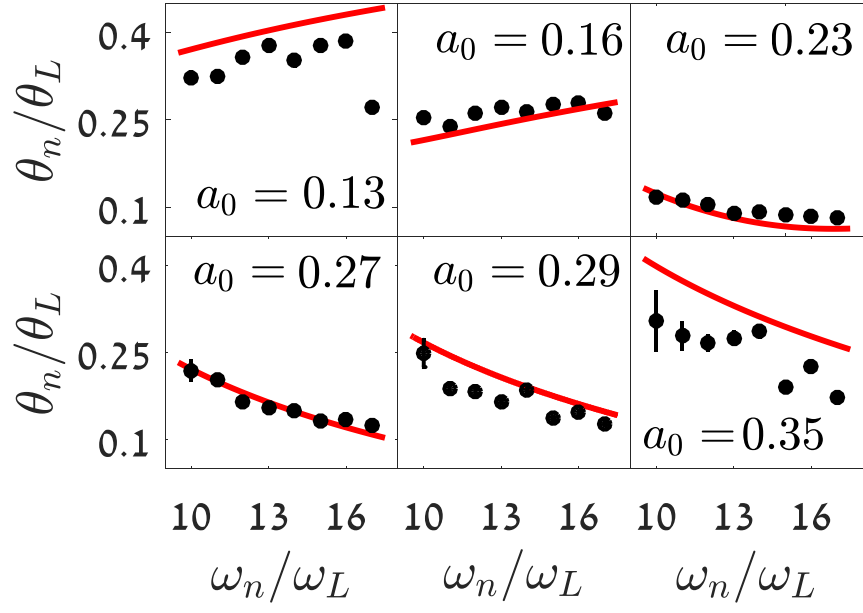

FIG. 6. The measured emission divergence vs harmonic order for different laser intensities (circles). The bars represent the uncertainty resulting from the Gaussian fitting process. The analytic predictions of our model (solid line) are calculated for the $a_{0}$ value indicated in each case.

ties. The curves were all calculated with the same values of $a_{i}$ and $L_{0}$, as used in Fig. 5 .

We validated our results using two-dimensional (2D) PIC simulations carried out with the EPOCH code [34]. The laser pulse in these simulations was $p$ polarized with $\lambda=800 \mathrm{~nm}$, with a pulse duration of $15 \mathrm{fs}$ FWHM and a waist width of $3.4 \lambda$ FWHM. The pulse impinges on an overdense plasma $\left(n_{0}=250 n_{c}\right)$ with an exponential density gradient:

$$
n\left(x_{\perp}, x_{\|}\right)=n_{0} \exp \left(-\frac{x_{\perp}}{L_{0}\left[a\left(x_{\|}\right)-a_{i}\right]}\right) .
$$

Here $x_{\perp}, x_{\|}$are the coordinates perpendicular and parallel to the target surface, respectively; $L_{0}=\lambda / 11.3$; and $a_{i}=$ 0.044 . The target was set at a $55^{\circ}$ angle of incidence. The simulations ran with a space step of $\Delta x=\lambda / 280,30$ particles per cell, and a box size of $35 \lambda \times 35 \lambda$.

We performed a series of simulations for laser intensities in the range of $0.15<a_{0}<0.40$. One example snapshot of the target-parallel component of the electric field at $a_{0}=0.2$, $40 \mathrm{fs}$ after the pulse reflection, is shown in Fig. 7. The harmonics (marked with white arrows) are visible as thin modulation

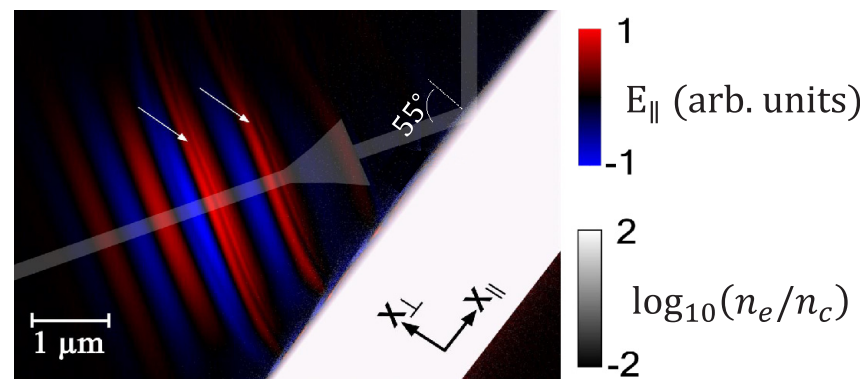

FIG. 7. PIC simulation results. A snapshot of the target-parallel component of the electric field, after reflection off the PM. XUV pulses (marked with white arrows) appear as thin modulations on the reflected field. 
in the field of the reflected pulse. We applied a numeric bandpass filter in the spectral range of $\mathrm{H} 5-\mathrm{H} 15$ to isolate the XUV front and extract its radius of curvature, from which the focusing parameter $\Psi$, and subsequently the harmonic beam divergence, were calculated according to Eq. (15). The results are indicated with red crosses in Fig. 5. The data and the PIC results are in very good agreement, except for the lowest simulated intensity.

In this Letter, we showed how excess divergence in CWE that originates from intrinsic intensity-dependent phase variations can be canceled out in particular conditions which result in a flat wavefront in the emission plane. CWE dynamics are one dimensional in nature, as can be shown by using a boost transformation to the normal-incidence frame [35]. Our extension of the CWE model does not alter this fundamental attribute, except that a different plasma scale is used at each point along the lateral direction.

Our results highlight the sensitivity of CWE to the early stage of plasma expansion, which is governed by the tempo- ral intensity profile and by laser absorption during the few hundred fs prior to the peak of the pulse. As suggested by Eqs. (6) and (14), for a fixed main-pulse duration the beam divergence may be controlled through the pedestal slope, e.g., by altering the ps contrast using an acousto-optic programmable dispersive filter [36]. This high sensitivity may explain the different degrees of beam divergence that were measured using different laser systems (Fig. 5), and the deviation of our model from the experimental data for $a_{0}<0.15$.

We acknowledge aid in target fabrication from the Tel Aviv University Center for Nanoscience and Nanotechnology. Simulations were performed using EPOCH, which was developed as part of the UK Engineering and Physical Sciences Research Council (EPSRC) funded Project No. EP/G054940/1. We acknowledge the support by the Pazy Foundation, Grant No. 27707241, and by the Zuckerman STEM Leadership Program.
[1] G. A. Mourou, T. Tajima, and S. V. Bulanov, Optics in the relativistic regime, Rev. Mod. Phys. 78, 309 (2006).

[2] R. Hörlein, B. Dromey, D. Adams, Y. Nomura, S. Kar, K. Markey, P. Foster, D. Neely, F. Krausz, G. D. Tsakiris et al., High contrast plasma mirror: Spatial filtering and second harmonic generation at $1019 \mathrm{~W} \mathrm{~cm}^{-2}$, New J. Phys. 10, 083002 (2008).

[3] B. Dromey, S. Kar, M. Zepf, and P. Foster, The plasma mirrora subpicosecond optical switch for ultrahigh power lasers, Rev. Sci. Instrum. 75, 645 (2004).

[4] S. A. Reed, T. Matsuoka, S. Bulanov, M. Tampo, V. Chvykov, G. Kalintchenko, P. Rousseau, V. Yanovsky, R. Kodama, D. W. Litzenberg et al., Relativistic plasma shutter for ultraintense laser pulses, Appl. Phys. Lett. 94, 201117 (2009).

[5] H. Vincenti, S. Monchocé, S. Kahaly, G. Bonnaud, P. Martin, and F. Quéré, Optical properties of relativistic plasma mirrors, Nat. Commun. 5, 3403 (2014).

[6] M. Thévenet, A. Leblanc, S. Kahaly, H. Vincenti, A. Vernier, F. Quéré, and J. Faure, Vacuum laser acceleration of relativistic electrons using plasma mirror injectors, Nat. Phys. 12, 355 (2015).

[7] P. H. Bucksbaum, The future of attosecond spectroscopy, Science 317, 766 (2007).

[8] A. P. Mancuso, T. Gorniak, F. Staier, O. M. Yefanov, R. Barth, C. Christophis, B. Reime, J. Gulden, A. Singer, M. E. Pettit et al., Coherent imaging of biological samples with femtosecond pulses at the free-electron laser FLASH, New J. Phys. 12, 035003 (2010).

[9] S. Mathias, H. C. Kapteyn, and M. M. Murnane, Ultrafast material science probed using coherent $\mathrm{x}$-ray pulses from high-harmonic generation BT-ultrafast nonlinear optics, in Ultrafast Nonlinear Optics (Springer International Publishing, Heidelberg, 2013), pp. 149-175.

[10] B. Dromey, M. Zepf, A. Gopal, K. Lancaster, M. S. Wei, K. Krushelnick, M. Tatarakis, N. Vakakis, S. Moustaizis, R. Kodama et al., High harmonic generation in the relativistic limit, Nat. Phys. 2, 456 (2006).
[11] D. an der Brügge and A. Pukhov, Enhanced relativistic harmonics by electron nanobunching, Phys. Plasmas 17, 033110 (2010).

[12] J. M. Mikhailova, M. V. Fedorov, N. Karpowicz, P. Gibbon, V. T. Platonenko, A. M. Zheltikov, and F. Krausz, Isolated Attosecond Pulses from Laser-Driven Synchrotron Radiation, Phys. Rev. Lett. 109, 245005 (2012).

[13] F. Quéré, C. Thaury, P. Monot, S. Dobosz, P. Martin, J.-P. Geindre, and P. Audebert, Coherent Wake Emission of HighOrder Harmonics from Overdense Plasmas, Phys. Rev. Lett. 96, 125004 (2006).

[14] C. Thaury, F. Quéré, J.-P. Geindre, A. Levy, T. Ceccotti, P. Monot, M. Bougeard, F. Réau, P. D’Oliveira, P. Audebert et al., Plasma mirrors for ultrahigh-intensity optics, Nat. Phys. 3, 424 (2007).

[15] A. A. Gonoskov, A. V. Korzhimanov, A. V. Kim, M. Marklund, and A. M. Sergeev, Ultrarelativistic nanoplasmonics as a route towards extreme-intensity attosecond pulses, Phys. Rev. E 84, 046403 (2011).

[16] M. R. Edwards and J. M. Mikhailova, The x-ray emission effectiveness of plasma mirrors: Reexamining power-law scaling for relativistic high-order harmonic generation, Sci. Rep. 10, 5154 (2020).

[17] F. Quéré, C. Thaury, J.-P. Geindre, G. Bonnaud, P. Monot, and P. Martin, Phase Properties of Laser High-Order Harmonics Generated on Plasma Mirrors, Phys. Rev. Lett. 100, 095004 (2008).

[18] M. Yeung, B. Dromey, D. Adams, S. Cousens, R. Hörlein, Y. Nomura, G. D. Tsakiris, and M. Zepf, Beaming of High-Order Harmonics Generated from Laser-Plasma Interactions, Phys. Rev. Lett. 110, 165002 (2013).

[19] F. Brunel, Not-So-Resonant, Resonant Absorption, Phys. Rev. Lett. 59, 52 (1987).

[20] C. Thaury and F. Quéré, High-order harmonic and attosecond pulse generation on plasma mirrors: Basic mechanisms, J. Phys. B: At., Mol. Opt. Phys. 43, 213001 (2010). 
[21] A. Malvache, A. Borot, F. Quéré, and R. Lopez-Martens, Coherent wake emission spectroscopy as a probe of steep plasma density profiles, Phys. Rev. E 87, 035101 (2013).

[22] A. Leblanc and F. Quéré, In situ ptychographic measurements of high-order harmonic sources from plasma mirrors: A theoretical and numerical study, Phys. Plasmas 25, 013112 (2018).

[23] A. Leblanc, S. Monchocé, H. Vincenti, S. Kahaly, J.-L. Vay, and F. Quéré, Spatial Properties of High-Order Harmonic Beams from Plasma Mirrors: A Ptychographic Study, Phys. Rev. Lett. 119, 155001 (2017)

[24] S. Kahaly, S. Monchocé, H. Vincenti, T. Dzelzainis, B. Dromey, M. Zepf, P. Martin, and F. Quéré, Direct Observation of Density-Gradient Effects in Harmonic Generation from Plasma Mirrors, Phys. Rev. Lett. 110, 175001 (2013).

[25] A. Leblanc, S. Monchocé, C. Bourassin-Bouchet, S. Kahaly, and F. Quéré, Ptychographic measurements of ultrahighintensity laser-plasma interactions, Nat. Phys. 12, 301 (2016).

[26] P. Mora, Collisionless expansion of a Gaussian plasma into a vacuum, Phys. Plasmas 12, 112102 (2005).

[27] A. Jullien, S. Kourtev, O. Albert, G. Cheriaux, J. Etchepare, N. Minkovski, and S. M. Saltiel, Highly efficient temporal cleaner for femtosecond pulses based on cross-polarized wave generation in a dual crystal scheme, Appl. Phys. B: Lasers Opt. 84, 409 (2006).

[28] A. Kessel, V. E. Leshchenko, O. Jahn, M. Krüger, A. Münzer, A. Schwarz, V. Pervak, M. Trubetskov, S. A. Trushin, F. Krausz et al., Relativistic few-cycle pulses with high contrast from picosecond-pumped OPCPA, Optica 5, 434 (2018).
[29] S. A. Self, Focusing of spherical Gaussian beams, Appl. Opt. 22, 658 (1983).

[30] E. Porat, A. Levanon, D. Roitman, I. Cohen, R. Louzon, and I. Pomerantz, Towards direct-laser-production of relativistic surface harmonics, in Relativistic Plasma Waves and Particle Beams as Coherent and Incoherent Radiation Sources III, edited by D. A. Jaroszynski and M. Hur (SPIE, Bellingham, WA, 2019), Vol. 11036, p. 17.

[31] Amplitude Tech, Sequoia HD, https://amplitude-laser.com/ add_ons/metrology/sequoia/.

[32] M. Bocoum, F. Böhle, A. Vernier, A. Jullien, J. Faure, and R. Lopez-Martens, Spatial-domain interferometer for measuring plasma mirror expansion, Opt. Lett. 40, 3009 (2015).

[33] A. Tarasevitch, A. Orisch, D. von der Linde, P. Balcou, G. Rey, J.-P. Chambaret, U. Teubner, D. Klöpfel, and W. Theobald, Generation of high-order spatially coherent harmonics from solid targets by femtosecond laser pulses, Phys. Rev. A 62, 023816 (2000).

[34] T. D. Arber, K. Bennett, C. S. Brady, A. Lawrence-Douglas, M. G. Ramsay, N. J. Sircombe, P. Gillies, R. G. Evans, H. Schmitz, A. R. Bell et al., Contemporary particle-in-cell approach to laser-plasma modelling, Plasma Phys. Controlled Fusion 57, 113001 (2015).

[35] A. Bourdier, Oblique incidence of a strong electromagnetic wave on a cold inhomogeneous electron plasma. Relativistic effects, Phys. Fluids 26, 1804 (1983).

[36] P. Tournois, Acousto-optic programmable dispersive filter for adaptive compensation of group delay time dispersion in laser systems, Opt. Commun. 140, 245 (1997). 